\title{
The Accurate Mass Formulas of Leptons, Quarks, Gauge Bosons, the Higgs Boson, and Cosmic Rays
}

\author{
Ding-Yu Chung \\ Utica, MI, USA \\ Email:dy_chung@yahoo.com
}

Received 21 July 2016; accepted 28 August 2016; published 31 August 2016

Copyright (C) 2016 by author and Scientific Research Publishing Inc.

This work is licensed under the Creative Commons Attribution International License (CC BY). http://creativecommons.org/licenses/by/4.0/

(c) (i) Open Access

\section{Abstract}

One of the biggest unsolved problems in physics is the particle masses of all elementary particles which cannot be calculated accurately and predicted theoretically. In this paper, the unsolved problem of the particle masses is solved by the accurate mass formulas which calculate accurately and predict theoretically the particle masses of all leptons, quarks, gauge bosons, the Higgs boson, and cosmic rays (the knees-ankles-toe) by using only five known constants: the number (seven) of the extra spatial dimensions in the eleven-dimensional membrane, the mass of electron, the masses of $Z$ and $W$ bosons, and the fine structure constant. The calculated masses are in excellent agreements with the observed masses. For examples, the calculated masses of muon, top quark, pion, neutron, and the Higgs boson are 105.55 MeV, 175.4 GeV, 139.54 MeV, $939.43 \mathrm{MeV}$, and 126 $\mathrm{GeV}$, respectively, in excellent agreements with the observed $105.65 \mathrm{MeV}, 173.3 \mathrm{GeV}, 139.57 \mathrm{MeV}$, 939.27 MeV, and $126 \mathrm{GeV}$, respectively. The mass formulas also calculate accurately the masses of the new particle at $750 \mathrm{GeV}$ from the LHC and the new light boson at $17 \mathrm{MeV}$. The theoretical base of the accurate mass formulas is the periodic table of elementary particles. As the periodic table of elements is derived from atomic orbitals, the periodic table of elementary particles is derived from the seven principal mass dimensional orbitals and seven auxiliary mass dimensional orbitals. All elementary particles including leptons, quarks, gauge bosons, the Higgs boson, and cosmic rays can be placed in the periodic table of elementary particles. The periodic table of elementary particles is based on the theory of everything as the computer simulation model of physical reality consisting of the mathematical computation, digital representation and selective retention components. The computer simulation model of physical reality provides the seven principal mass dimensional orbitals and seven auxiliary mass dimensional orbitals for the periodic table of elementary particles.

\section{Keywords}

Mass Formulas, Particle Masses, Leptons, Quarks, Gauge Bosons, Higgs Boson, Cosmic Rays, 
The Periodic Table of Elementary Particles, Computer Simulation, Knees-Ankles-Toe, The Theory of Everything

\section{Introduction}

According to Johan Hansson, one of the ten biggest unsolved problems in physics [1] is the incalculable particle masses of leptons, quarks, gauge bosons, and the Higgs boson. The Standard Model of particle physics contains the particles masses of leptons, quarks, and gauge bosons which cannot be calculated or predicted theoretically. From a theoretical point of view, the particle mass is a total unsolved problem and they might as well have been random numbers drawn from a hat. The repetition of leptons and quarks with increasing masses has also remained as unsolved problem. Max Jammer [2] concluded that nobody knows what particle masses really are. The mass parameters experimentally measured for elementary particles have no theoretical explanation whatsoever. From the vantage point of theory, the masses could just as well be a set of randomly generated numbers.

This paper provides a solution to the unsolved problem of particle masses. In this paper, all elementary particles and cosmic rays (the knees-ankles-toe) can be calculated accurately and predicted theoretically by the accurate mass formulas of leptons, quarks, gauge bosons, the Higgs boson, and cosmic rays by using only five known constants: the number (seven) of the extra spatial dimensions in the eleven-dimensional membrane, the mass of electron, the masses of $\mathrm{Z}$ and $\mathrm{W}$ bosons, and the fine structure constant. The calculated masses are in excellent agreements with the observed masses. For examples, the calculated masses of muon, top quark, pion, neutron, and the Higgs boson are $105.55 \mathrm{MeV}, 175.4 \mathrm{GeV}, 139.54 \mathrm{MeV}, 939.43 \mathrm{MeV}$, and $126 \mathrm{GeV}$, respectively, in excellent agreements with the observed $105.65 \mathrm{MeV}, 173.3 \mathrm{GeV}, 139.57 \mathrm{MeV}, 939.27 \mathrm{MeV}$, and 126 $\mathrm{GeV}$, respectively. The theoretical base of the accurate mass formulas is the periodic table of elementary particles [3]-[6]. As the periodic table of elements is derived from atomic orbitals, the periodic table of elementary particles is derived from the seven principal mass dimensional orbitals and seven auxiliary mass dimensional orbitals. All elementary particles including leptons, quarks, gauge bosons, the Higgs boson, and cosmic rays can be placed in the periodic table of elementary particles.

The periodic table of elementary particles is derived from the computer simulation model of physical reality [7] which is the theory of everything [8]. We are living in a computer simulation to simulate physical reality which has the same computer simulation process as virtual reality (computer-simulated reality). The computer simulation process involves the digital representation of data, the mathematical computation of the digitized data in geometric formation and transformation in space-time, and the selective retention of events in a narrative. Conventional physics cannot explain physical reality clearly, while computer-simulated physics can explain physical reality clearly by using the computer simulation process consisting of the digital representation component, the mathematical computation component, and the selective retention component. The computer simulation model of physical reality provides the seven principal mass dimensional orbitals and seven auxiliary mass dimensional orbitals for the periodic table of elementary particles.

In Section 2, the periodic table of elementary particles is derived from the computer simulation of physical reality. Section 3 deals with the gauge boson mass formula and the cosmic ray mass formula. Section 4 explains the lepton mass formula and the quark mass formula. Section 5 describes the Higgs boson mass formula.

\section{The Periodic Table of Elementary Particles}

The periodic table of elementary particles is derived from the computer simulation model of physical reality consisting of the mathematical computation, digital representation, and selective retention components.

\subsection{The Mathematical Computation Component}

The geometry in the mathematic computation component for the computer simulation process is oscillating M-theory. M-theory with eleven-dimensional membrane is an extension of string theory with ten-dimensional string, in contrast to the observed 4D. In conventional M-theory, space-time dimensional number (D) is fixed. As a result, the observed 4D results from the compactization of the extra space dimensions in 11D M-theory, However, there is no experimental proof for compactized extra space dimensions, and there are numerous ways for 
the compactization of the extra space dimensions [9]. As described before [7], the geometry for the mathematical computation is oscillating M-theory derived from oscillating membrane-string-particle whose space-time dimension number oscillates between 11D and 10D and between 10D and 4D dimension by dimension reversibly. There is no compactization. Matters in oscillating M-theory include 11D membrane $\left(2_{11}\right)$ as membrane (denoted as 2 for 2 space dimensions) in $11 \mathrm{D}, 10 \mathrm{D}$ string $\left(1_{10}\right)$ as string (denoted as 1 for 1 space dimension) in $10 \mathrm{D}$, and variable $\mathrm{D}$ particle $\left(0_{4}\right.$ to 11$)$ as particle (denoted as 0 for 0 space dimension) in $4 \mathrm{D}$ to $11 \mathrm{D}$.

As described previously [10], the QVSL (quantum varying speed of light) transformation transforms both spacetime dimension number (D) and mass dimension number (d). In the QVSL transformation, the decrease in the speed of light leads to the decrease in space-time dimension number and the increase of mass in terms of increasing mass dimension number from 4 to 10 ,

$$
\begin{gathered}
c_{\mathrm{D}}=c / \alpha^{\mathrm{D}-4}, \\
E=M_{0} \cdot\left(c^{2} / \alpha^{2(\mathrm{D}-4)}\right) \\
=\left(M_{0} / \alpha^{2(\mathrm{~d}-4)}\right) \cdot c^{2} . \\
c_{\mathrm{D}}=c_{\mathrm{D}-n} / \alpha^{2 n}, \\
M_{0, \mathrm{D}, \mathrm{d}}=M_{0, \mathrm{D}-n, \mathrm{~d}+n} \alpha^{2 n}, \\
\mathrm{D}, \mathrm{d} \stackrel{\mathrm{QVSL}}{\longrightarrow}(\mathrm{D} \mp n),(\mathrm{d} \pm n) \\
E_{\text {vacuum }, \mathrm{D}}=E-M_{0, \mathrm{D}} c^{2},
\end{gathered}
$$

where $c_{\mathrm{D}}$ is the quantized varying speed of light in space-time dimension number, $\mathrm{D}$, from 4 to $10 ; c$ is the observed speed of light in the 4D space-time; $\alpha$ is the fine structure constant for electromagnetism, $\mathrm{E}$ is energy; $M_{0}$ is rest mass; $\mathrm{D}$ is the space-time dimension number from 4 to $10 ; \mathrm{d}$ is the mass dimension number from 4 to $10 ; n$ is an integer; and $E_{\text {vacuum }}=$ vacuum energy. For example, in the QVSL transformation, a particle with 10D4d is transformed to a particle with 4D10d from Equation (1f). Calculated from Equation (1e), the rest mass of 4D10d is $1 / \alpha^{12}$ $\approx 137^{12}$ times of the mass of 10D4d. In terms of rest mass, $10 \mathrm{D}$ space-time has $4 \mathrm{~d}$ with the lowest rest mass, and 4D space-time has $10 \mathrm{~d}$ with the highest rest mass. Rest mass decreases with increasing space-time dimension number. The decrease in rest mass means the increase in vacuum energy $\left(E_{\text {vacuum, D }}\right)$, so vacuum energy increases with increasing space-time dimension number. The vacuum energy of $4 \mathrm{D}$ particle is zero from Equation $(1 \mathrm{~g})$. The mass dimension number is limited from 4 to 10 , because $4 \mathrm{D}$ is the minimum space-time, and 11D membrane and $10 \mathrm{D}$ string are equal in the speed of light, rest mass, and vacuum energy. Since the speed of light for $>4 \mathrm{D}$ particle is greater than the speed of light for $4 \mathrm{D}$ particle, the observation of $>4 \mathrm{D}$ superluminal particles by $4 \mathrm{D}$ particles violates casualty. Thus, $>4 \mathrm{D}$ particles are hidden particles with respect to $4 \mathrm{D}$ particles. Particles with different space-time dimensions are transparent and oblivious to one another, and separate from one another if possible.

In the digital representation component for the computer simulation process, data in physical reality are represented by digital representations. Both data and digital representations exist. For the digital representation component of physical reality, the three intrinsic data (properties) are rest mass-kinetic energy, electric charge, and spin which are represented by the digital space structure, the digital spin, and the digital electric charge, respectively.

\subsection{Digital Space Structure}

The digital representations of rest mass and kinetic energy are 1 as attachment space for the space of matter and 0 as detachment space for the zero-space of matter, respectively [7] [11]. In the digital space structure, attachment space attaches to matter permanently or reversibly. Detachment space detaches from the object at the speed of light. Attachment space relates to rest mass and reversible movement, while detachment space relates to irreversible kinetic energy.

As shown previously [8], our universe is the dual asymmetrical positive-energy-negative-energy universe where the positive-energy universe on attachment space absorbed the interuniversal void on detachment space to result in the combination of attachment space and detachment space. The combination of $n$ units of attachment space as 1 and $n$ 
units of detachment space as 0 brings about three different digital space structures: binary partition space, miscible space, or binary lattice space as below.

$$
(1)_{n} \quad+\quad(0)_{n} \quad \stackrel{\text { combination }}{\longrightarrow}(1)_{n}(0)_{n}, \quad(1+0)_{n}, \quad \text { or } \quad(10)_{n}
$$

attachment space detachment space binary partition space, miscible space, binary lattice space

Binary partition space, $(1)_{n}(0)_{n}$, consists of two separated continuous phases of multiple quantized units of attachment space and detachment space. In miscible space, $(1+0)_{n}$, attachment space is miscible to detachment space, and there is no separation of attachment space and detachment space. Binary lattice space, $\left(\begin{array}{ll}1 & 0\end{array}\right)_{n}$, consists of repetitive units of alternative attachment space and detachment space. Binary partition space, miscible space, and binary lattice space relate to quantum mechanics, special relativity, and force fields, respectively [7].

Bounias and Krasnoholovets [12] propose that the reduction of dimension can be done by slicing dimension, such as slicing 3 space dimension object (block) into infinite units of 2 space dimension objects (sheets). As shown previously [8], the positive-energy 10D4d particle universe as our observable universe with high vacuum energy was transformed into the $4 \mathrm{D} 10 \mathrm{~d}$ universe with zero vacuum energy at once, resulting in the inflation. During the Big Bang following the inflation, the $10 \mathrm{~d}$ (mass dimension) particle in attachment space denoted as 1 was sliced by detachment space denoted as 0 . For example, the slicing of $10 \mathrm{~d}$ particle into $4 \mathrm{~d}$ particle is as follows.

$$
1_{10} \stackrel{\text { slicing }}{\longrightarrow} 1_{4} \quad \sum_{\mathrm{d}=5}^{10}\left(0_{4} 1_{4}\right)_{n, \mathrm{~d}}
$$

$10 \mathrm{~d}$ particle $4 \mathrm{~d}$ core particle binary lattice space

where $1_{10}$ is $10 \mathrm{~d}$ particle; $1_{4}$ is $4 \mathrm{~d}$ particle; $\mathrm{d}$ is the mass dimension number of the dimension to be sliced; $n$ as the number of slices for each dimension; and $\left(0_{4} 1_{4}\right)_{n}$ is binary lattice space with repetitive units of alternative $4 \mathrm{~d}$ attachment space and $4 \mathrm{~d}$ detachment space. For $4 \mathrm{~d}$ particle starting from $10 \mathrm{~d}$ particle, the mass dimension number of the dimension to be sliced is from $d=5$ to $d=10$. Each mass dimension is sliced into infinite quantized units $(n=\infty)$ of binary lattice space, $\left(0_{4} 1_{4}\right)_{\infty}$. For $4 d$ particle, the $4 d$ core particle is surrounded by 6 types (from $\mathrm{d}=5$ to $d=10$ ) of infinite quantized units of binary lattice space. Such infinite quantized units of binary lattice space represent the infinite units $(n=\infty)$ of separate virtual orbitals in a gauge force field, while the dimension to be sliced is "mass dimensional orbital" (DO), representing a type of gauge force field. In addition to the six DO's for gauge force fields from $d=5$ to $d=10$, gravity appears as the seventh DO at $d=11$. As a result, there are seven mass dimensional orbitals as in Figure 1.

\subsection{The Digital Spin}

The digital representations of the exclusive and the inclusive occupations of positions are $1 / 2$ spin fermion and integer spin boson, respectively [7]. Fermions, such as electrons and protons, follow the Pauli exclusion principle which excludes fermions of the same quantum-mechanical state from being in the same position. Bosons, such as photons or helium atoms, follow the Bose-Einstein statistics which allows bosons of the same quantum-mechanical state being in the same position. As a result, the digital representations of the exclusive and the inclusive occupations of positions are 1/2 spin fermion and integer spin boson, respectively. The symmetry between fermion and boson is supersymmetry. Two supersymmetry transformations from boson to fermion and from fermion to boson yield a spatial translation. In physical reality, supersymmetry is varying supersymmetry [8]. In varying supersymmetry, the repetitive transformation between fermion and boson brings about a spatial translation and the transformation into the adjacent mass dimension number. Varying supersymmetry transformation is one of the two steps in transformation involving the oscillation between 10D particle and 4D particle. The transformation during the oscillation between 10D particle and 4D particle involves the stepwise two-step transformation consisting of the QVSL transformation and the varying supersymmetry transformation. The QVSL

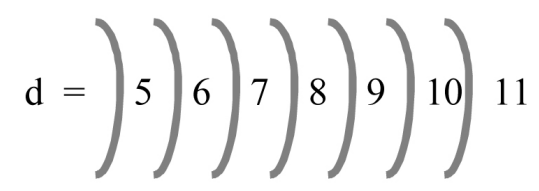

Figure 1. The seven mass dimensions as mass dimensional orbitals. 
transformation involves the transformation of space-time dimension, D whose mass increases with decreasing D for the decrease in vacuum energy. The varying supersymmetry transformation involves the transformation of the mass dimension number, $\mathrm{d}$ whose mass decreases with decreasing $\mathrm{d}$ for the fractionalization of particle, as follows.

stepwise two-step varying transformation

(1) $\mathrm{D}, \mathrm{d} \stackrel{\mathrm{QVSL}}{\longleftrightarrow}(\mathrm{D} \mp 1),(\mathrm{d} \pm 1)$

(2) $\mathrm{D}, \mathrm{d} \stackrel{\text { varying supersymmetry }}{\longleftrightarrow} \mathrm{D},(\mathrm{d} \pm 1)$

The repetitive stepwise two-step transformation between 10D4d and 4D4d as follows.

$$
10 \mathrm{D} 4 \mathrm{~d} \leftrightarrow \text { 9D5d } \leftrightarrow \text { 9D4d } \leftrightarrow 8 \mathrm{D} 5 \mathrm{~d} \leftrightarrow \cdots \leftrightarrow 4 \mathrm{D} 5 \mathrm{~d} \leftrightarrow 4 \mathrm{D} 4 \mathrm{~d}
$$

In this two-step transformation, the transformation from 10D4d to 9D5d involves the QVSL transformation as in Equation (1d). Calculated from Equation (1e), the mass of 9D5d is $1 / \alpha^{2} \approx 137^{2}$ times of the mass of 10D4d. The transformation of 9D5d to 9D4d involves the varying supersymmetry transformation. In the normal supersymmetry transformation, the repeated application of the fermion-boson supersymmetry transformation carries over a boson (or fermion) from one point to the same boson (or fermion) at another point at the same mass. In the varying supersymmetry transformation, the repeated application of the fermion-boson supersymmetry transformation carries over a boson from one point to the boson at another point at different mass dimension number in the same space-time number. The repeated varying supersymmetry transformation carries over a boson $B_{d}$ into a fermion $F_{d}$ and a fermion $F_{d}$ to a boson $B_{d-1}$, which can be expressed as follows

$$
\begin{aligned}
& M_{\mathrm{d}, \mathrm{F}}=M_{\mathrm{d}, \mathrm{B}} \alpha_{\mathrm{d}, \mathrm{B}}, \\
& M_{\mathrm{d}-1, \mathrm{~B}}=M_{\mathrm{d}, \mathrm{F}} \alpha_{\mathrm{d}, \mathrm{F}},
\end{aligned}
$$

where $M_{\mathrm{d}, \mathrm{B}}$ and $M_{\mathrm{d}, \mathrm{F}}$ are the masses for a boson and a fermion, respectively; $\mathrm{d}$ is the mass dimension number; and $\alpha_{\mathrm{d}, \mathrm{B}}$ or $\alpha_{\mathrm{d}, \mathrm{F}}$ is the fine structure constant that is the ratio between the masses of a boson and its fermionic partner. Assuming $\alpha$ 's are the same, it can be expressed as

$$
M_{\mathrm{d}, \mathrm{B}}=M_{\mathrm{d}+1, \mathrm{~B}} \alpha_{\mathrm{d}+1}^{2} .
$$

\subsection{The Digital Electric Charge}

As described before [13], the digital representations of the allowance and the disallowance of irreversible kinetic energy are integral electric charges and fractional electric charges, respectively. Individual integral charge with irreversible kinetic energy to cause irreversible movement is allowed, while individual fractional charge with irreversible kinetic energy is disallowed. The disallowance of irreversible kinetic energy for individual fractional charge brings about the confinement of individual fractional charges to restrict the irreversible movement resulted from kinetic energy. Collective fractional charges are confined by the short-distance confinement force field where the sum of the collective fractional charges is integer. As a result, fractional charges are confined and collective. The confinement force field includes gluons in QCD (quantum chromodynamics) for collective fractional charge quarks in hadrons and the magnetic flux quanta for collective fractional charge quasiparticles in the fractional quantum Hall effect (FQHE) [14]-[16].

In the periodic table of elementary particles, fractional charge quarks have their own seven mass dimensional orbitals as the seven auxiliary mass dimensional orbitals in addition to the seven principal mass dimensional orbitals for leptons as in Figure 2.

\subsection{The Selective Retention Component}

The selective retention component retains selectively events in a narrative. The retained events are unified by the common narrative. The narrative of physical reality is the four-stage evolution of our cyclic dual universe. The four force fields are unified by the four-stage evolution.

Our dual universe is the globally reversible cyclic dual universe as shown in Figure 3 [7] for the evolution of our universe as described previously [7].

The four reversible stages in the globally reversible cyclic dual universe are: 1) the formation of the 11D 


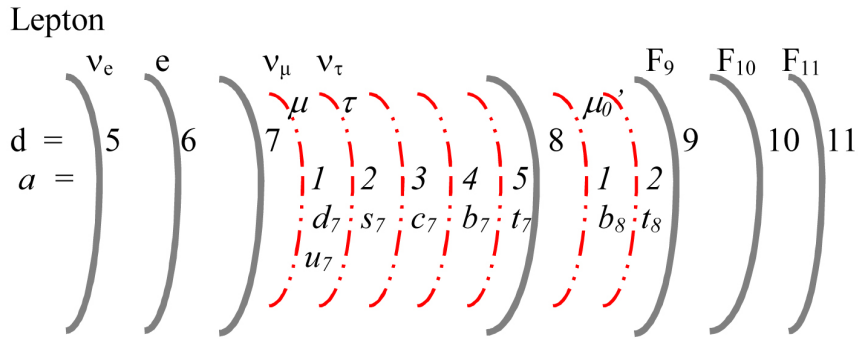

Quark

Figure 2. Leptons and quarks in the seven principal dimensional orbitals (solid lines) denoted by the principal dimensional orbital number $\mathrm{d}$ and the seven auxiliary dimensional orbitals (dash-dotted lines) denoted by the auxiliary dimensional number $a$.

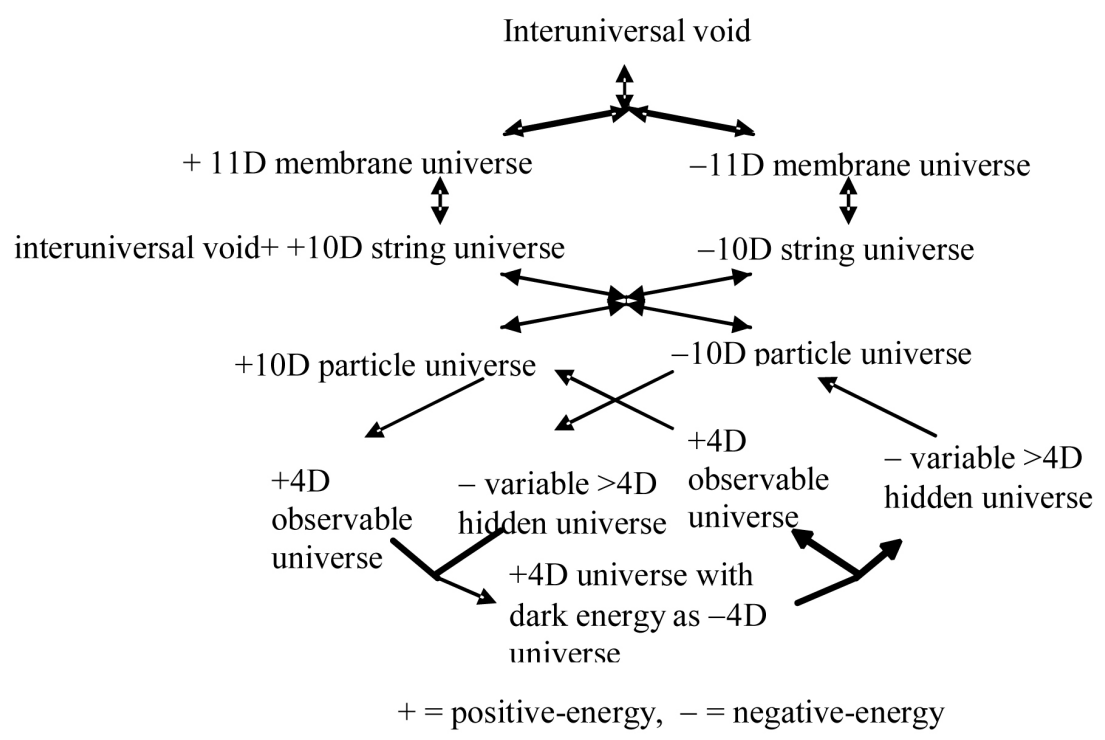

Figure 3. The globally reversible cyclic dual universe.

membrane dual universe; 2) the formation of the 10D string dual universe; 3 ) the formation of the 10D particle dual universe; and 4) the formation of the asymmetrical dual universe. The pre-strong force (the prototype of observed strong force) and pre-gravity emerged in the stage 2 . The pre-electromagnetism emerged in the stage 3 , while the weak force emerged in the stage 4 . The selective retention of the four force fields provides the four force fields (gauge bosons) for the four of the seven principal mass dimensional orbitals $\left(\mathrm{B}_{\mathrm{d}}\right)$ as in Table 1 . The other three of the seven principal mass dimensional orbitals are for the $\mathrm{CP}$ (right) nonconservation, the $\mathrm{CP}$ (left) nonconservation, and the weak (right) force.

The periodic table of elementary particles for leptons, quarks, and gauge boson is in Table 2 with both the principal mass dimensional orbital numbers and the auxiliary mass dimensional orbital numbers.

\section{The Gauge Boson Mass Formula and the Cosmic Ray Mass Formula}

The gauge boson mass formula is Equation (7) derived from Equation (6c) based on the digital spin structure,

$$
M_{\mathrm{d}+1, \text { gauge boson }}=M_{\mathrm{d}, \text { gaugeboson }} / \alpha_{\mathrm{d}+1}^{2} .
$$

Each dimension has its own $\alpha_{\mathrm{d}}$, and all $\alpha_{\mathrm{d}}$ 's except $\alpha_{7}\left(\alpha_{w}\right)$ of the seventh dimension (weak interaction) are equal to $\alpha$, the fine structure constant of electromagnetism. The lowest energy boson is the Coulombic field for electromagnetism based on Equation (6b) and the second lowest boson energy is $\pi_{1 / 2}$ (a spin 1 boson as a half of the spin 0 pion) with the mass of $70 \mathrm{MeV}$ for the strong interaction. As described in Section 4, this boson $\mathrm{B}_{6}$ is used to construct gluon. 
Table 1. The masses of the principal mass dimensional orbitals (gauge bosons) $\alpha=\alpha_{\mathrm{e}}, \mathrm{d}=$ mass dimensional orbital number.

\begin{tabular}{ccccc}
\hline $\mathrm{B}_{\mathrm{d}}$ & $M_{\mathrm{d}}$ & $\mathrm{GeV}$ (calculated) & Gauge boson & Interaction \\
\hline $\mathrm{B}_{5}$ & $M_{e} \alpha$ & $3.7 \times 10^{-6}$ & $\mathrm{~A}=$ photon & Electromagnetic \\
$\mathrm{B}_{6}$ & $M_{e} / \alpha$ & $7 \times 10^{-2}$ & $\pi_{1 / 2}$ & Strong \\
$\mathrm{B}_{7}$ & $M_{6} / \alpha_{w}{ }^{2}$ & 91.1876 (given) & $Z_{L}^{0}$ & weak (left) \\
$\mathrm{B}_{8}$ & $M_{7} / \alpha^{2}$ & $1.71 \times 10^{6}$ & $\mathrm{X}_{\mathrm{R}}$ & CP (right) nonconservation \\
$\mathrm{B}_{9}$ & $M_{8} / \alpha^{2}$ & $3.22 \times 10^{10}$ & $\mathrm{X}_{\mathrm{L}}$ & $\mathrm{CP}$ (left) nonconservation \\
$\mathrm{B}_{10}$ & $M_{9} / \alpha^{2}$ & $6.04 \times 10^{14}$ & $Z_{R}^{0}$ & weak (right) \\
$\mathrm{B}_{11}$ & $M_{10} / \alpha^{2}$ & $1.13 \times 10^{19}$ & $\mathrm{G}$ & Gravity \\
\hline
\end{tabular}

Table 2. The periodic table of elementary particles $\mathrm{d}=$ principal mass dimensional orbital number, a = auxiliary mass dimensional orbital number.

\begin{tabular}{|c|c|c|c|c|c|c|c|c|c|}
\hline d & $a=0$ & 1 & 2 & 1 & 2 & 3 & 4 & 5 & \\
\hline & Lepton & & & Quark & & & & & Boson \\
\hline 5 & $v_{\mathrm{e}}$ & & & & & & & & $\mathrm{B}_{5}=\mathrm{A}$ \\
\hline 6 & e & & & & & & & & $\mathrm{B}_{6}=\pi_{1 / 2}$ \\
\hline 7 & & $v_{\mu} \mu$ & $v_{t} / \tau$ & $\mathrm{d}_{7} / \mathrm{u}_{7}$ & $\mathrm{~s}_{7}$ & $\mathrm{c}_{7}$ & $\mathrm{~b}_{7}$ & $\mathrm{t}_{7}$ & $\mathrm{~B}_{7}=Z_{L}^{0}$ \\
\hline 8 & & $\mu_{0}^{\prime}$ (hidden) & & $\mathrm{b}_{8}$ (hidden) & $\mathrm{t}_{8}$ & & & & $\mathrm{~B}_{8}=\mathrm{X}_{\mathrm{R}}$ \\
\hline 9 & $\mathrm{~F}_{9}$ & & & & & & & & $\mathrm{~B}_{9}=\mathrm{X}_{\mathrm{L}}$ \\
\hline 10 & $\mathrm{~F}_{10}$ & & & & & & & & $\mathrm{~B}_{10}=Z_{R}^{0}$ \\
\hline 11 & $\mathrm{~F}_{11}$ & & & & & & & & $B_{11}$ gravity \\
\hline
\end{tabular}

$$
\begin{gathered}
M_{5, \mathrm{~B}}=\alpha M_{6, \mathrm{~F}}=\alpha M_{\mathrm{e}} . \\
M_{\mathrm{B}_{6}}=M_{\mathrm{B}_{5}} / \alpha^{2}=M_{\mathrm{e}} / \alpha=M_{\pi_{1 / 2}}
\end{gathered}
$$

In Table $1, \alpha_{w}$ is not same as $\alpha$ because there is a mixing between electromagnetism and the weak interaction in the standard theory of the electroweak interaction, and $\sin \theta_{w}$ is not equal to 1 where $\theta_{w}$ is the weak mixing angle [17]. As a result, $\alpha_{w}$ instead of $\alpha$ is used to calculate $M_{\mathrm{B}_{7}}$ from $M_{\mathrm{B}_{6}}$ based on the gauge boson formula Equation (7). $M_{\mathrm{B}_{7}}$ is the mass of weak $\mathrm{Z}$ boson $M_{\mathrm{Z}}$ which is $91.1876 \mathrm{GeV}$.

$$
\begin{aligned}
& M_{\mathrm{B}_{7}}=M_{\mathrm{Z}}=M_{\mathrm{B}_{6}} / \alpha_{w}^{2} \\
& \alpha_{w}=\sqrt{M_{\mathrm{B}_{6}} / M_{\mathrm{Z}}}
\end{aligned}
$$

The calculated value for $\alpha_{w}$ is 0.02771 . As described in the following paragraphs and Table $3, \mathrm{~B}_{8}, \mathrm{~F}_{9}$ and $\mathrm{B}_{9}$ in Table 2 are observed in cosmic rays as the first knee, the second knee, and the toe, respectively, where the calculated masses are in good agreement with the observed masses [6]. The calculated energy for $\mathrm{B}_{11}$ is $1.13 \times 10^{19} \mathrm{GeV}$ in good agreement with the Planck mass, $1.22 \times 10^{19} \mathrm{GeV}$ for gravity.

High-energy cosmic rays which have much higher energies than the energy of particles accelerated by the Large Hadron Collider provide the study of elementary particles beyond the capacity of particle accelerators. The cosmic ray mass formula is to the energy spectrum for the knees-ankles-toe of cosmic rays [6]. The energy spectrum from $10^{9} \mathrm{eV}$ to $10^{20} \mathrm{eV}$ appears to follow a single power law except few breaks at the knees-ankles-toe [18]. The power index increases at the first knee and the second knee, and decreases at the ankle. Above $4 \times 10^{19} \mathrm{eV}$, the power index increases as the "toe". The fine structure of the cosmic ray spectrum [19] shows that an ankle with decrease in power index is in between the first knee and the second knee, resulting in two knees, two ankles, and one toe. As explained previously [6], the knees-ankles-toe are explained by the very high-energy fermions and bosons in the periodic table of elementary particles. In the periodic table, some high-energy dimensional fermions $\left(F_{d}\right.$ where $d=$ dimen- 
Table 3. The calculated masses for dimensional bosons-fermions and the observed $\mathrm{eV}$ for the knees-ankles-toe. $\mathrm{B}_{\mathrm{d}}=$ mass dimensional boson, $\mathrm{F}_{\mathrm{d}}=$ mass dimensional fermion.

\begin{tabular}{ccccc}
\hline $\mathrm{B}_{\mathrm{d}}, \mathrm{F}_{\mathrm{d}}$ & calculated eV & Calculation & cosmic rays & observed eV \\
\hline $\mathrm{B}_{8}$ & $1.7 \times 10^{15}$ & & the first knee & $3 \times 10^{15}$ \\
the midpoint between $\mathrm{B}_{8}$ and $\mathrm{F}_{9}$ & $2 \times 10^{16}$ & Equation $(11)$ & the first ankle & $2 \times 10^{16}$ \\
$\mathrm{~F}_{9}$ & $2.4 \times 10^{17}$ & $M_{\mathrm{B}_{8}} / \alpha$ & the second knee & $3 \times 10^{17}$ \\
the midpoint between $\mathrm{F}_{9}$ and $\mathrm{B}_{9}$ & $2.8 \times 10^{18}$ & Equation $(11)$ & the second ankle & $3 \times 10^{18}$ \\
$\mathrm{~B}_{9}$ & $3.2 \times 10^{19}$ & $M_{\mathrm{F}_{9}} / \alpha$ & the toe & beyond the GZK limit \\
$\mathrm{F}_{10}$ & $4.4 \times 10^{21}$ & $M_{\mathrm{B}_{9}} / \alpha$ & not observed \\
\hline
\end{tabular}

sional orbital number from 5 to 11$)$ and bosons $\left(B_{d}\right)$ are involved in the knees-ankles-toe. At the knees and the toe, some parts of the energies from the energy sources of cosmic rays are spent to generate $F_{d}$ and $B_{d}$, resulting in the increase of power index. The ankles are the the middle points (midpoints) between the adjacent dimensional fermions and bosons. At a midpoint, the energy is too high to keep the thermally unstable high- energy dimensional particle, resulting in the decay and the decrease of power index.

The cosmic ray mass formula is derived from Equations (6a) and (6b)

$$
\begin{aligned}
& M_{\mathrm{d}, \mathrm{F}}=M_{\mathrm{d}, \mathrm{B}} \alpha \\
& M_{\mathrm{d}-1, \mathrm{~B}}=M_{\mathrm{d}, \mathrm{F}} \alpha
\end{aligned}
$$

where $M_{\mathrm{d}, \mathrm{B}}$ and $M_{\mathrm{d}, \mathrm{F}}$ are the masses for a boson and a fermion, respectively; $\mathrm{d}$ is the mass dimension number from 8 to 10 ; and $\alpha_{\mathrm{d}, \mathrm{B}}$ or $\alpha_{\mathrm{d}, \mathrm{F}}$ is the fine structure constant. The midpoint is expressed as follows.

$$
M_{\text {midpoint }}=\exp \left(\left(\ln \left(M_{\text {adjacent dimensional ferion }}\right)+\ln \left(M_{\text {adjacent dimensional boson }}\right)\right) / 2\right)
$$

The calculated masses of $\mathrm{B}_{8}$, the midpoint, $\mathrm{F}_{9}$, the midpoint, and $\mathrm{B}_{9}$, are $1.7 \times 10^{15}, 2 \times 10^{16}, 2.4 \times 10^{17}, 2.8 \times$ $10^{18}$, and $3.2 \times 10^{19} \mathrm{eV}$, respectively, which are in good agreement with observed $3 \times 10^{15}, 2 \times 10^{16}, 3 \times 10^{17}, 3 \times$ $10^{18}$, and $4 \times 10^{19} \mathrm{eV}$ for the first knee, the first ankle, the second knee, the second ankle, and the toe, respectively as in Table 3 .

The mass of $\mathrm{F}_{10}$ is $4.4 \times 10^{21} \mathrm{eV}$ beyond the GZK (Greisen-Zatsepin-Kuzmin) limit, which occurs at about $5 \times$ $10^{19} \mathrm{eV}$, as the maximum energy of cosmic ray particles that have traveled long distances (about 160 million light years), due to the theoretical energy losses of higher-energy ray particles and to scattering from photons in the cosmic microwave background. Therefore, $\mathrm{F}_{10}$ and above are not observed.

\section{The Lepton Mass Formula and the Quark Mass Formula}

The lepton mass formula and the quark mass formula are derived from the electric digital charge structure where the digital representations of the allowance and the disallowance of irreversible kinetic energy are integral electric charges and fractional electric charges, respectively [13]. The collectivity of fractional charges requires the attachment of energy as flux quanta to bind fractional charges. As a result, the integer-fraction transformation from integral charges to fractional charges involves the integer-fraction transformation to incorporate flux quanta similar to the composite fermion theory for the FQHE [20] [21]. In the composite fermion model, the formation of composite fermion is through the attachment of an even number of magnetic flux quanta to electron, while the formation of composite boson is through the attachment of an odd number of magnetic flux quanta to electron. In the same way, the integer-fraction transformation from integral charges to fractional charges for quarks consists of the three steps: 1) the attachment of an even number of flux quanta to individual integral charge fermions to form individual integral charge composite fermions $\left.\left(F_{c}\right) ; 2\right)$ the attachment of an odd number of flux quanta to individual integral charge composite fermions to form transitional collective integral charge composite bosons $\left(\mathrm{B}_{\mathrm{c}}\right)$; and 3$)$ the conversion of flux quanta into the confinement force field to confine collective fractional charge composite quarks $\left(\mathrm{Q}_{\mathrm{c}}\right)$ converted from composite bosons as follows. 
individual $\mathrm{e} \overline{\mathrm{e}} \stackrel{2 \text { flux quanta }}{\longrightarrow}$ individual $\mathrm{F}_{\mathrm{c}} \overline{\mathrm{F}}_{\mathrm{c}} \stackrel{3 \text { flux quanta }}{\longrightarrow}$

transitional collective $\mathrm{B}_{\mathrm{c}} \overline{\mathrm{B}}_{\mathrm{c}} \stackrel{\mathrm{QCD}}{\longrightarrow}$

collective $2 \mathrm{Q}_{\mathrm{c}} \overline{\mathrm{Q}}_{\mathrm{c}}$ of $\pm \frac{1}{3}$ and $\pm \frac{2}{3}$ charge with 3 gluons + individual $\overline{\mathrm{e}}$

where $\mathrm{F}_{\mathrm{c}}, \mathrm{B}_{\mathrm{c}}$, and $\mathrm{Q}_{\mathrm{c}}$ are the composite fermion the composite boson, and the composite quark, respectively. The first step of the integer-fraction transformation from electron to quark is the attachment of 2 flux quanta to individual integral charge electrons to form individual composite fermions $\left(\mathrm{F}_{\mathrm{c}}\right.$ 's). The flux quanta $(70.0252 \mathrm{MeV})$ are the flux quanta as proposed by Peter Cameron to calculate accurately the masses of pion, muon, and proton [22]. The quantum of $70.0252 \mathrm{MeV}$ is also the bosonic mass quantum proposed by Malcolm H. MacGregor for a basic building block to calculate accurately the masses of hadrons [23]. According the periodic table of elementary particles, the flux quantum is $\mathrm{B}_{6}$ from Table 1. From Equation (6b), the relationship between $\mathrm{B}_{6}$ and electron $\left(\mathrm{F}_{5}\right)$ is as follows.

$$
M_{\mathrm{B}_{6}}=M_{\mathrm{F}_{5}} / \alpha=M_{\mathrm{e}} / \alpha=70.0252 \mathrm{MeV}
$$

where $\alpha$ is the fine structure constant for electromagnetism. The $\mathrm{F}_{\mathrm{pc}}$ (the principal composite fermion) consists of two $\mathrm{B}_{6}$ as flux quanta.

$$
M_{\mathrm{F}_{\mathrm{pc}}}=2 M_{\mathrm{B}_{6}}=140.0505 \mathrm{MeV}
$$

The mass of pion (boson) is the mass of the principal composite fermion $\left(\mathrm{F}_{\mathrm{pc}}\right)$ minus the mass of electron (fermion) [22].

$$
M_{\pi}=M_{\mathrm{F}_{\mathrm{pc}}}-M_{\mathrm{e}}=139.5395 \mathrm{MeV}
$$

which is in excellent agreement with the observed $139.5702 \mathrm{MeV}$.

In the second step for the attachment of odd number of flux quanta, the odd number of flux quanta can be one flux quantum for one principal composite fermion or three flux quanta for three principal composite fermions, resulting in lepton or quark, respectively. For the formation of lepton, the second step is the attachment of one flux quantum to one individual integral charge principal composite fermion to form the transitional integral charge principal composite boson. In the second step, the principal composite boson for composite lepton is $\mathrm{B}_{6}+$ $\mathrm{F}_{\mathrm{pc}}$. In the third step, the principal composite boson is converted into two composite leptons $\left(\mathrm{L}_{\mathrm{c}}\right)$ with the addition of electrons, resulting in muon as follows.

$$
\begin{gathered}
M_{\mathrm{B}_{\mathrm{pc}}} \text { for } \mathrm{L}_{\mathrm{c}}=M_{\mathrm{F}_{\mathrm{pc}}}+M_{\mathrm{B}_{6}}=2 M_{\mathrm{B}_{6}}+M_{\mathrm{B}_{6}}=3 M_{\mathrm{B}_{6}} \\
M_{\mathrm{L}_{\mathrm{c}}}=M_{\mu}=M_{\mathrm{e}}+M_{\mathrm{B}_{\mathrm{pc}}} / 2=M_{\mathrm{e}}+3 M_{\mathrm{B}_{6}} / 2=M_{e}+\frac{3 M_{e}}{2 \alpha}=105.5491 \mathrm{MeV}
\end{gathered}
$$

which is in excellent agreement with the observed $105.6584 \mathrm{MeV}$. The muon mass formula in Equation (16b) is identical to the Barut lepton mass formula for muon [24]. Equation (16b) explains the origin of $3 / 2$ which cannot be explained easily by Cameron, MacGregor, and Barut. Without binding the composite leptons together by the flux quanta, leptons have integral electric charges instead of fractional electric charges.

The Barut lepton mass formula [24] is expressed as follows.

$$
M_{\text {lepton }}=M_{\mathrm{e}}+\frac{3 M_{\mathrm{e}}}{2 \alpha} \sum_{a=0}^{n} a^{4},
$$

where $n=0,1$, and 2 are for $\mathrm{e}, \mu$, and $\tau$, respectively. The calculated mass of $\tau$ is $1786.2 \mathrm{MeV}$ in good agreement with the observed mass as $1776.82 \mathrm{MeV}$. According to Barut, the second term, $\sum_{a=0}^{n} a^{4}$ of the mass formula is for the BohrSommerfeld quantization for a charge-dipole interaction in a circular orbit. The experimental proof of this dipole-interaction in a circular orbit is shown as the light boson at $17 \mathrm{MeV}$ from the generation of pairs of electrons and positrons by firing protons at thin targets of lithium-7 [25] [26]. The light boson is the orbit $\mathrm{e}_{\mathrm{o}} \overline{\mathrm{e}}_{\mathrm{o}}$ in the circular orbit. The masses of the bosons from orbit $\mathrm{e}_{\mathrm{o}} \overline{\mathrm{e}}_{\mathrm{o}}$ are calculated as follows. 


$$
M_{\text {boson }}=M_{\mathrm{e}_{\mathrm{o}} \overline{\mathrm{e}}_{\mathrm{o}}}=2 M_{\mathrm{e}_{\mathrm{o}}}=2 M_{\mathrm{e}} \sum_{a=1}^{n} a^{4}
$$

where the masses of the bosons for $n=1$ and 2 are $2 M_{\mathrm{e}}$ for e $\overline{\mathrm{e}}$ (electron-positron pair) and $34 M_{\mathrm{e}}$ for the light boson, respectively. The calculated mass of the light boson is $17.374 \mathrm{MeV}$ in excellent agreement with the observed mass as $17 \mathrm{MeV}$ [25]. As in Figure 2 and Table 2, $v_{\mu}$ and $v_{\tau}$ share the same orbitals with $\mu$ and $\tau$, respectively. All neutrinos $\left(v_{e}, v_{\mu}\right.$ and $\left.v_{\tau}\right)$ in the periodic table of elementary particles (Table 2$)$ are nearly massless because of chiral symmetry (permanent left-handed).

For the formation of quark, the second step is the attachment of 3 flux quanta ( $\mathrm{B}_{6}$ 's) to three individual integral charge principal composite fermions $\left(\mathrm{F}_{\mathrm{pc}}\right.$ 's) to form the transitional collective integral charge principal composite bosons $\left(\mathrm{B}_{\mathrm{pc}}\right.$ 's). The transitional principal composite bosons are derived from the combination of the three principal composite fermions $\left(3 \mathrm{~F}_{\mathrm{pc}}\right.$ 's) with the three flux quanta $\left(3 \mathrm{~B}_{6}\right.$ 's) which are connected and located at the same position in the same $3-\mathrm{F}_{\mathrm{pc}}$ energy level. One $3-\mathrm{F}_{\mathrm{pc}}$ energy level consists of the three connected $\mathrm{F}_{\mathrm{pc}}$ sites with the connected three flux quanta $\left(3 \mathrm{~B}_{6}\right.$ 's). The mass of the transitional principal composite bosons $\mathrm{B}_{\mathrm{pc}}$ is as follows.

$$
M_{\mathrm{B}_{\mathrm{pc}}}=3 M_{\mathrm{F}_{\mathrm{pc}}}+3 M_{\mathrm{B}_{6}}=6 M_{\mathrm{B}_{6}}+3 M_{\mathrm{B}_{6}}=9 M_{\mathrm{B}_{6}}=9 M_{\mathrm{e}} / \alpha=630.227 \mathrm{MeV}
$$

In the third step, 3 flux quanta ( $\mathrm{B}_{6}$ 's) are converted to 3-color gluons (red, green, and blue) in QCD. Each of the three $F_{p c}$ sites in the energy level has $\pm 1 / 3$ charge. The fractional charges of quarks are the integer multiples of $\pm 1 / 3 \mathrm{e}$. One principal composite boson $\left(\mathrm{B}_{\mathrm{pc}}\right)$ is converted into two composite quarks (fermions) in the same way as the conversion of one photon (boson) into two fermions (electron-positron). As a result, the principal composite quark $\left(\mathrm{Q}_{\mathrm{pc}}\right)$ has $1 / 2$ mass of the principal composite boson $\left(\mathrm{B}_{\mathrm{pc}}\right)$ in addition to the mass of $1 / 3$ and $2 / 3$ electrons for the three $\mathrm{F}_{\mathrm{c}}$ sites for different electric charges as follows.

$$
M_{\mathrm{Q}_{\mathrm{pc}}}=\frac{1 \text { or } 2 M_{\mathrm{e}}}{3}+\frac{M_{\mathrm{B}_{\mathrm{pc}}}}{2}=\frac{1 \text { or } 2 M_{\mathrm{e}}}{3}+\frac{9 M_{\mathrm{e}}}{2 \alpha}=315.28 \text { or } 315.45 \mathrm{MeV}
$$

From Equation (20), the principal composite quark with $1 / 3$ electric charge is the principal composite $d$ quark with $315.28 \mathrm{MeV}$, and the principal composite quark with $2 / 3$ charge is the principal composite u quark with 315.45 MeV.

According to the periodic table of elementary particles (Table 2), quarks have the auxiliary mass dimensional orbital in addition to the principal mass dimensional orbital as in Figure 2. As a result, there are the auxiliary composite quarks $\left(\mathrm{Q}_{\mathrm{ac}}\right)$ in addition to the principal composite quarks $\left(\mathrm{Q}_{\mathrm{pc}}\right)$. The three-step transformation in the integer-fraction transformations from integral charge to fractional charge is applicable to the auxiliary composite quarks. The auxiliary flux quantum is $B_{a}$ which is in between $d_{7}$ and $d_{8}$ as shown in Figure 2. As a result, $\alpha_{w}$ instead of $\alpha$ is used for $\mathrm{B}_{\mathrm{a} 7}$. Muon, a composite lepton, instead of electron is used for $\mathrm{B}_{\mathrm{a} 7}$ as follows.

$$
M_{\mathrm{B}}=M_{\mu} \alpha_{w}
$$

where $\alpha_{w}$ is the fine structure constant for weak force from Equation (9). In the first step, the $\mathrm{F}_{\mathrm{ac}}$ (the auxiliary composite fermion) consists of two $\mathrm{B}_{\mathrm{a} 7}$ as auxiliary flux quanta.

$$
M_{\mathrm{F}_{\mathrm{ac}}}=2 M_{\mathrm{B}_{\mathrm{a} 7}}=2 M_{\mu} \alpha_{w}
$$

The second step is the attachment of 3 auxiliary flux quanta $\left(\mathrm{B}_{\mathrm{a} 7}\right.$ 's) to the individual integral charge auxiliary composite fermions $\left(\mathrm{F}_{\mathrm{ac}}\right.$ 's) to form the transitional collective integral charge auxiliary composite bosons $\left(\mathrm{B}_{\mathrm{ac}}\right.$ 's). The transitional auxiliary composite bosons are derived from the combination of the three auxiliary composite fermions $\left(3 \mathrm{~F}_{\mathrm{ac}}\right.$ 's) with the three flux quanta $\left(3 \mathrm{~B}_{\mathrm{a} 7}\right.$ 's) which are connected and located at the same position in the same 3-F $\mathrm{F}_{\mathrm{ac}}$ energy level. One 3- $\mathrm{F}_{\mathrm{ac}}$ energy level consists of the three connected $\mathrm{F}_{\mathrm{ac}}$ sites with the connected three auxiliary flux quanta $\left(3 \mathrm{~B}_{\mathrm{a}}\right.$ 's). The mass of the transitional auxiliary composite bosons $\mathrm{B}_{\mathrm{ac}}$ is as follows.

$$
M_{\mathrm{B}_{\mathrm{ac}}}=3 M_{\mathrm{F}_{\mathrm{ac}}}+3 M_{\mathrm{B}_{\mathrm{ac} 7}}=6 M_{\mathrm{B}_{\mathrm{ac} 7}}+3 M_{\mathrm{B}_{\mathrm{ac} 7}}=9 M_{\mathrm{B}_{\mathrm{ac} 7}}=9 M_{\mu} \alpha_{w}
$$

In the third step, 3 auxiliary flux quanta ( $\mathrm{B}_{\mathrm{a}}$ 's) are converted to 3-color gluons (red, green, and blue) in $\mathrm{QCD}$ to confine the collective fractional charge auxiliary composite quarks $\left(\mathrm{Q}_{\mathrm{ac}}\right.$ 's) conversed from the transitional auxiliary composite bosons $\left(\mathrm{B}_{\mathrm{ac}}\right.$ 's). One composite boson $\left(\mathrm{B}_{\mathrm{ac}}\right)$ is converted into two composite quarks (fermions) in the same way as the conversion of one photon (boson) into two fermions (electron-positron). As a re- 
sult, the auxiliary composite quark $\left(\mathrm{Q}_{\mathrm{ac}}\right)$ has $1 / 2$ mass of the auxiliary composite boson $\left(\mathrm{B}_{\mathrm{ac}}\right)$.

$$
M_{\mathrm{Q}_{\mathrm{ac}}}=M_{\mathrm{B}_{\mathrm{ac}}} / 2=9 M_{\mu} \alpha_{w} / 2=13.16 \mathrm{MeV}
$$

The composite quark mass formula is the combination of the principal composite quark and the auxiliary composite quark with the Bohr-Sommerfeld quantization for a charge-dipole interaction in a circular orbit as follows.

$$
M_{\mathrm{Q}_{\mathrm{c}}}=M_{\mathrm{Q}_{\mathrm{pc}}}+M_{\mathrm{Q}_{\mathrm{ac}}} \sum_{a=1}^{n} a^{4}=\frac{1 \text { or } 2 M_{\mathrm{e}}}{3}+\frac{9 M_{\mathrm{e}}}{2 \alpha}+\frac{9 M_{\mu} \alpha_{w}}{2} \sum_{a=1}^{n} a^{4}
$$

where $n=1,2,3,4$, and 5 for $\mathrm{u} / \mathrm{d}, \mathrm{s}, \mathrm{c}, \mathrm{b}$, and a part of $\mathrm{t}$, respectively.

At $n=5$ for Equation (25), the mass $(140.4 \mathrm{GeV})$ is greater than $\mathrm{B}_{7}(91.1876 \mathrm{GeV})$ as in Table 1 , so the last two auxiliary mass dimensional orbitals of the seven auxiliary dimensional orbitals are in between $\mathrm{d}_{8}$ and $\mathrm{d}_{9}$. The formation of the auxiliary mass dimensional orbitals between $d_{8}$ and $d_{9}$ requires the extra-principal flux quantum as $\mathrm{B}_{7}$ instead of $\mathrm{B}_{6}$. For the first step in the three-step transformation from integer charge to fractional charge for the extra quarks is to form the extra composite fermion. $\mathrm{B}_{7}$ is $\mathrm{Z}$ boson. The $\mathrm{F}_{\mathrm{epc}}$ (the extra principal composite fermion) consists of two $\mathrm{B}_{7}$ as flux quanta. The $\mathrm{F}_{\mathrm{epc}}$ (the extra principal composite fermion) consists of two $\mathrm{B}_{7}$ as flux quanta.

$$
M_{\mathrm{F}_{\mathrm{epc}}}=2 M_{\mathrm{B}_{7}}=2 M_{\mathrm{Z}}
$$

For the second step to form the extra principal composite boson, only one extra-principal flux quantum is added to one extra-principal composite fermion, because the 3-color principal electric and 3-color auxiliary flux quanta already exist, and there is no need for three extra-principal flux quanta.

$$
M_{\mathrm{B}_{\mathrm{epc}}}=M_{\mathrm{F}_{\mathrm{epc}}}+M_{\mathrm{B}_{7}}=2 M_{\mathrm{Z}}+M_{\mathrm{Z}}=3 M_{\mathrm{Z}}
$$

The third step is the conversion of the extra-principal composite boson to two extra-principal quarks $\left(\mathrm{Q}_{\mathrm{epc}}\right)$. Only The neutral $\mathrm{B}_{\mathrm{epc}}$ is used for $\mathrm{Q}_{\mathrm{epc}}$, so no electron is added.

$$
M_{\mathrm{Q}_{\mathrm{epc}}}=M_{\mathrm{B}_{\mathrm{epc}}} / 2=3 M_{\mathrm{Z}} / 2=136.78 \mathrm{GeV}
$$

Since $\mathrm{Q}_{\mathrm{epc}}$ involves only one extra-principal flux quantum, $\mathrm{Q}_{\mathrm{epc}}$ is identical to the extra-composite lepton $\mathrm{L}_{\mathrm{ec}}$ which is neutral extra-muon $\mu_{0}^{\prime}$.

$$
M_{\mu_{0}^{\prime}}=M_{\mathrm{Q}_{\mathrm{epc}}}=3 M_{\mathrm{Z}} / 2=136.78 \mathrm{GeV}
$$

The extra-auxiliary flux quantum is $\mathrm{B}_{\mathrm{ea}}$ is like Equation (21).

$$
M_{\mathrm{B}_{\mathrm{ea}}}=M_{\mu_{0}^{\prime}} \alpha
$$

where the fine structure constant in between $\mathrm{d}_{8}$ and $\mathrm{d}_{9}$ is $\alpha$. For the first step in the three-step transformation from integer charge to fractional charge, the extra-auxiliary composite fermion $\left(\mathrm{F}_{\text {eac }}\right)$ is the composite of two $\mathrm{B}_{8 \mathrm{ea}}$ 's.

$$
M_{\mathrm{F}_{\mathrm{ea}}}=2 M_{\mathrm{B}_{8 \mathrm{ea}}}=2 M_{\mu_{0}^{\prime}} \alpha
$$

For the second step to form the extra-auxiliary composite boson $\mathrm{B}_{\mathrm{eac}}$, only one extra-auxiliary flux quantum is needed in the same way for the formation of the extra-principal composite boson.

$$
M_{\mathrm{B}_{\text {eac }}}=M_{\mathrm{F}_{\mathrm{eac}}}+M_{\mathrm{B}_{8 \mathrm{ea}}}=2 M_{\mathrm{B}_{8 \mathrm{ea}}}+M_{\mathrm{B}_{8 \mathrm{ea}}}=3 M_{\mathrm{B}_{8 \mathrm{ea}}}=3 M_{\mu_{0}^{\prime}} \alpha
$$

For the third step to form quark from composite boson $\mathrm{B}_{\mathrm{eac}}$, extra-auxiliary composite boson is converted into two extra-auxiliary composite quarks $\left(\mathrm{Q}_{\mathrm{eac}}\right)$.

$$
M_{\mathrm{Q}_{\mathrm{eac}}}=M_{\mathrm{B}_{\mathrm{eac}}} / 2=3 M_{\mu_{0}^{\prime}} \alpha / 2=3\left(3 M_{\mathrm{Z}} / 2\right) \alpha / 2=9 M_{\mathrm{Z}} \alpha / 4
$$

The mass formula for the extra- composite quark $\left(\mathrm{Q}_{\mathrm{ec}}\right)$ with the Bohr-Sommerfeld quantization for a charge-dipole interaction in a circular orbit is as follows.

$$
M_{\mathrm{Q}_{\mathrm{ec}}}=M_{\mathrm{Q}_{\mathrm{epc}}}+M_{\mathrm{Q}_{\mathrm{eac}}} \sum_{a^{\prime}=1}^{n^{\prime}} a^{\prime 4}=\frac{3 M_{Z}}{2}+\frac{9 M_{Z} \alpha}{4} \sum_{a^{\prime}=1}^{n^{\prime}} a^{\prime 4}
$$


where $n^{\prime}=1$ and 2 for $\mathrm{b}$ and $\mathrm{t}$, respectively.

Quark is the combination of the composite quark from Equation (25) and the extra-composite quark from Equation (34). The quark mass formula is as follows.

$$
M_{\text {quark }}=M_{\mathrm{Q}_{\mathrm{c}}}+M_{\mathrm{Q}_{\mathrm{ec}}}=\frac{1 \text { or } 2 M_{\mathrm{e}}}{3}+\frac{9 M_{\mathrm{e}}}{2 \alpha}+\frac{9 M_{\mu} \alpha_{w}}{2} \sum_{a=1}^{n} a^{4}+\frac{3 M_{\mathrm{Z}}}{2}+\frac{9 M_{\mathrm{Z}} \alpha}{4} \sum_{a^{\prime}=1}^{n^{\prime}} a^{\prime 4}
$$

where $n=1,2,3,4$, and 5 for $\mathrm{d} / \mathrm{u}$. $\mathrm{s}, \mathrm{c}, \mathrm{b}$, and $\mathrm{t}$, respectively; and $n^{\prime}=1$ and 2 for $\mathrm{b}$ and $\mathrm{t}$ respectively. The calculated masses for d, u, s, c, b, and t are 328.4 MeV, 328.6 MeV, 539 MeV, 1605.3 MeV, 4974.6 MeV, and 175.4 $\mathrm{GeV}$, respectively. In the Standard Model, there are three generations of leptons. Extra-muon $\mu_{0}^{\prime}$ is outside of the three generations of leptons in the Standard Model, so $\mu_{0}^{\prime}$ is hidden as shown in Table 2. As shown in Table 2, to be symmetrical to the hidden $\mu_{0}^{\prime}$, the extra composite quark, $\left(\mathrm{Q}_{\mathrm{epc}}\right)$ and the extra auxiliary composite quark $\left(Q_{\text {eac }}\right)$ for b quark are also hidden (absent).

The calculated mass of top quark is $175.4 \mathrm{GeV}$ in good agreement with the observed $173.3 \mathrm{GeV}$. The calculated masses are the constituent masses which include all different types of the flux quanta $\left(\mathrm{B}_{6}, \mathrm{~B}_{7 \mathrm{a}}\right.$, $\mathrm{B}_{8}$, and $\mathrm{B}_{8 \mathrm{ea}}$ ). The calculated constituent masses are comparable to the quark masses proposed by De Rujula, Georgi, and Glashow [27], Griffiths [28], and El Naschie [29]. The masses of hadrons are the combinations of the constituent quark masses minus the binding energy in the hadronic bond among quarks [30] [31]. The hadronic bond is the overlapping of the auxiliary dimensional orbitals, so it involves the auxiliary composite quarks, consisting of the primary auxiliary composite quark $\left(\mathrm{Q}_{\mathrm{ac}}\right)$ from Equation (24), the secondary auxiliary composite quark $\left(\mathrm{Q}_{\mathrm{ac}}^{\prime}\right)$, and the tertiary auxiliary composite quark $\left(\mathrm{Q}_{\mathrm{ac}}^{\prime \prime}\right)$. The secondary auxiliary composite quark $\left(\mathrm{Q}_{\mathrm{ac}}^{\prime}\right)$ is generated from the primary auxiliary composite quark in the same way as Equation (24).

$$
M_{\mathrm{Q}_{\mathrm{ac}}^{\prime}}=9 M_{\mathrm{Q}_{\mathrm{ac}}} \alpha_{w} / 2=1.64 \mathrm{MeV}
$$

The tertiary auxiliary composite quark $\left(\mathrm{Q}_{\mathrm{ac}}^{\prime \prime}\right)$ is generated from the secondary auxiliary composite quark in the same way as Equation (36).

$$
M_{\mathrm{Q}_{\mathrm{ac}}^{\mathrm{a}}}=9 M_{\mathrm{Q}_{\mathrm{ac}}^{\prime}} \alpha_{w} / 2=0.20 \mathrm{MeV}
$$

For neutron, the binding energy $\left(\mathrm{E}_{\mathrm{Q}-\mathrm{Q}}\right)$ in the hadronic bond between quarks involves the primary auxiliary composite quark to become the binding energy and the secondary auxiliary composite quark to become the mass to replace the primary auxiliary composite quark as below.

$$
E_{\mathrm{Q}-\mathrm{Q}}=2\left(M_{\mathrm{Q}_{\mathrm{ac}}}-M_{\mathrm{Q}_{\mathrm{ac}}^{\prime}}\right)=23.04 \mathrm{MeV}
$$

The mass of neutron which has two hadronic bonds is the sum of the constituent masses of $u, d$, and d quarks minus the binding energy from the two hadronic bonds.

$$
M_{\mathrm{N}}=M_{\mathrm{u}}+2 M_{\mathrm{d}}-2 E_{\mathrm{Q}-\mathrm{Q}}=939.43 \mathrm{MeV}
$$

The calculated mass of neutron is in good agreement with the observed value $939.57 \mathrm{MeV}$.

Proton is more stable than neutron, so it involves the additional binding energy from the tertiary auxiliary composite quark as below.

$$
E_{\mathrm{Q}-\mathrm{Q}}=2\left(M_{\mathrm{Q}_{\mathrm{ac}}}-M_{\mathrm{Q}_{\mathrm{ac}}^{\prime}}+M_{\mathrm{Q}_{\mathrm{ac}}^{\prime \prime}}\right)=23.45 \mathrm{MeV}
$$

The mass of proton which has two hadronic bonds is the sum of the constituent masses of $\mathrm{u}$, $\mathrm{u}$, and d quarks minus the binding energy from the two hadronic bonds.

$$
M_{\mathrm{P}}=2 M_{\mathrm{u}}+M_{\mathrm{d}}-2 E_{\mathrm{Q}-\mathrm{Q}}=938.78 \mathrm{MeV}
$$

The calculated mass of proton is in good agreement with the observed value $938.21 \mathrm{MeV}$.

Another way to form hadrons is through the combinations of $M_{\mathrm{e}} / \alpha(=70.03 \mathrm{MeV})$ and $3 M_{\mathrm{e}} / 2 \alpha(=105.04$ $\mathrm{MeV}$ ) as the mass quanta (mass building blocks) by the MacGregor's constituent quark model [23] [32]. Therefore, the masses of hadrons can be calculated by the combinations of the constituent quark masses from the quark mass formula as Equation (35) and by the combinations of the mass quanta from the MacGregor's constituent quark model [23] [30]-[32]. Another type of quark mass is the current mass which is much lower than the constituent mass, because it does not include some parts of principal composite quark $\left(Q_{\mathrm{pc}}\right)$ and auxiliary com- 
posite quark $\left(Q_{\mathrm{ac}}\right)$ which become massless gluon [28] [33].

Another extra-muon is charge extra-muon, $\mu_{ \pm}^{\prime}$, derived from $\mathrm{W}^{ \pm}$boson. The formation of $\mu_{ \pm}^{\prime}$ is same as $\mu_{0}^{\prime}$ based on Equations (28) and (29). (The observed mass of W boson is $80.385 \mathrm{GeV}$.)

$$
M_{\mu_{ \pm}^{\prime}}=3 M_{\mathrm{W}} / 2=120.58 \mathrm{GeV}
$$

Extra-muon $\mu^{\prime}$ includes neutral $\mu_{0}^{\prime}$ and charge $\mu_{ \pm}^{\prime}$.

\section{The Higgs Boson Mass Formula}

In the conventional model, under spontaneous symmetry breaking, zero-energy ground state space turns into the nonzero-energy scalar Higgs Field which exists permanently in the universe. The problem with such nonzero-energy field is the cosmological constant problem from the huge gravitational effect by the nonzero-energy Higgs field [34]. The coupling of massless particle to the Higgs field produces the transitional nonzero-energy Higgs field-particle composite which under spontaneous symmetry restoring produces the massive particle with the longitudinal component on zero-energy ground state space without the Higgs field as follows.

$$
\begin{aligned}
& \text { zerro-energy groud state space } \stackrel{\text { spontaneous symmetry breaking }}{\longrightarrow} \text { nonzero-energy scalar Higgs field } \\
& \stackrel{\text { massless particle }}{\longrightarrow} \text { [the transitional nonzero-energy Higgs field-particle composite }] \stackrel{\text { spontaneous symmetry restoring }}{\longrightarrow} \\
& \text { massive particle with the longitudinal component on zero-energy ground state space without the Higgs field }
\end{aligned}
$$

To avoid the cosmological problem from the huge gravitational effect by the nonzero-energy Higgs field is to make the Higgs field a transitional field which exists momentarily and to make zero-energy ground state space a permanent zero-energy ground state space which exists permanently in the universe [8]. For the digital space structure, such zero-energy ground state space is zero-energy attachment space which attaches particles to account for the longitudinal component, mass, and reversible movement. Unlike the conventional model, attachment space actively couples to massless particle. Under spontaneous symmetry breaking, the coupling of massless particle to zero-energy attachment space produces the transitional nonzero-energy Higgs field-particle composite which under spontaneous symmetry restoring produces massive particle on zero-energy attachment space with the longitudinal component without the Higgs field as follows.

$$
\begin{aligned}
& \text { massless particle }+ \text { zero-energy attachment space } \stackrel{\text { spontaneous symmetry breaking }}{\longrightarrow} \\
& \text { [the transitional non-zero energy Higgs field-particle composite] } \underset{\text { spontaneous symmetry restoring }}{\longrightarrow} \\
& \text { massive particle with the longitudinal component on zero-energy attachment space without the Higgs field }
\end{aligned}
$$

The opposite of attachment space is zero-energy detachment space which detaches particles to account for irreversible kinetic energy. Unlike the conventional model, detachment space actively couples to massive particle. Under spontaneous symmetry breaking, the coupling of massive particle to zero-energy detachment space produces the transitional nonzero-energy reverse Higgs field-particle composite which under spontaneous symmetry restoring produces massless particle on zero-energy detachment space without the longitudinal component without the reverse Higgs field as follows.

$$
\begin{aligned}
& \text { massive particle + zero-energy detachment space } \stackrel{\text { spontaneous symmetry breaking }}{\longrightarrow} \\
& \underset{\text { [the transitional nonzero-energy reverse Higgs field-particle composite] }}{\stackrel{\text { spontaneous symmetry restoring }}{\longrightarrow} \text { massless particle without the longitudinal component }} \\
& \text { on zero-energy detachment space without the reverse Higgs field }
\end{aligned}
$$

For the electroweak interaction in the Standard model where the electromagnetic interaction and the weak interaction are combined into one symmetry group, under spontaneous symmetry breaking, the coupling of the massless weak $\mathrm{W}$, weak Z, and electromagnetic A (photon) bosons to zero-energy attachment space produces the transitional nonzero-energy Higgs fields-bosons composites which under partial spontaneous symmetry restoring produce massive $\mathrm{W}$ and $\mathrm{Z}$ bosons on zero-energy attachment space with the longitudinal component without the Higgs field, massless A (photon), and massive Higgs boson as follows. 
massless WZ + zero-energy WZattachment space + massless A + zero-energy A attachment space A

$\stackrel{\text { spontaneous symmetry breaking }}{\longrightarrow}$ [the transitional nonzero-energy WZ Higgs field -WZ composite]

$+[$ nonzero-energy A Higgs field -A composite $] \stackrel{\text { partial spontaneous symmetry restoring }}{\longrightarrow}$

massive WZ with the longitudinal component on attachment space without the Higgs field

+ massless $\mathrm{A}+$ the nonzero energy massive Higgs boson

Being outside of the three-generation lepton-quark in the Standard Model, the Higgs boson adopts the extramuon condensate $\mu^{\prime} \bar{\mu}^{\prime}$ which is outside of the three-generation lepton-quark. In other words, the extra-muon condensate becomes the Avatar Higgs boson [5]. The extra-muon condensate $\mu^{\prime} \bar{\mu}^{\prime}$ includes $\mu_{0}^{\prime} \bar{\mu}_{0}^{\prime}$ and $\mu_{ \pm}^{\prime} \bar{\mu}_{ \pm}^{\prime}$. The extra-muon condensate composite consists of $\mu_{0}^{\prime} \bar{\mu}_{0}^{\prime}, \mu_{+}^{\prime} \bar{\mu}_{+}^{\prime}$, and $\mu_{-}^{\prime} \bar{\mu}_{-}^{\prime}$. From Equations (29) and (42), the mass of the $\mu^{\prime} \bar{\mu}^{\prime}$ condensate composite as the Higgs boson composite is as follows.

$$
\begin{aligned}
M_{\text {Higgs boson composite }} & =M_{\mu_{0}^{\prime} \bar{\mu}_{0}^{\prime}}+M_{\mu_{+}^{\prime} \bar{\mu}_{+}^{\prime}}+M_{\mu_{-}^{\prime} \bar{\mu}_{-}^{\prime}} \\
& =2(136.78) \mathrm{GeV}+2(120.58) \mathrm{GeV}+2(120.58) \mathrm{GeV} \\
& =756 \mathrm{GeV}
\end{aligned}
$$

This extra-muon condensate composite as the Higgs boson composite at $750 \mathrm{GeV}$ is in good agreement with the $756 \mathrm{GeV}$ diphoton excess observed from the Large Hadron Collider (LHC) with zero charge and zero spin [35] [36]. The $\mu^{\prime} \bar{\mu}^{\prime}$ condensate composite decays into three $\mu^{\prime} \bar{\mu}^{\prime}$ condensates. Just as the observed top quark is a bare quark with the observed mass of about $173 \mathrm{GeV}$ instead of about $346 \mathrm{GeV}$ (two times $173 \mathrm{GeV}$ ) for top quark-antitop quark boson, the observed mass of $\mu^{\prime} \bar{\mu}^{\prime}$ as the Higgs boson is the mass of bare $\mu^{\prime}$ as follows.

$$
M_{\text {observed } \mu^{\prime} \bar{\mu}^{\prime} \text { as the Higgsboson }}=M_{\mu^{\prime} \bar{\mu}^{\prime} \text { composite }} / 6=756 \mathrm{GeV} / 6=126 \mathrm{GeV}
$$

The calculated mass $(126 \mathrm{GeV})$ is in excellent agreement with the observed $125 \mathrm{GeV}$ [37] or $126 \mathrm{GeV}$ [38].

\section{Conclusions}

One of the biggest unsolved problems in physics is the particle masses of all elementary particles which cannot be calculated accurately and predicted theoretically. In this paper, the unsolved problem of the particle masses is solved by the accurate mass formulas which calculate accurately and predict theoretically the particle masses of all leptons, quarks, gauge bosons, the Higgs boson, and cosmic rays (the knees-ankles-toe) by using only five known constants: the number (seven) of the extra spatial dimensions in the eleven-dimensional membrane, the mass of electron, the masses of $\mathrm{Z}$ and $\mathrm{W}$ bosons, and the fine structure constant. The calculated masses are in excellent agreements with the observed masses. For examples, the calculated masses of muon, top quark, pion, neutron, and the Higgs boson are 105.55 MeV, 175.4 GeV, $139.54 \mathrm{MeV}, 939.43 \mathrm{MeV}$, and $126 \mathrm{GeV}$, respectively, in excellent agreements with the observed 105.65 MeV, 173.3 GeV, 139.57 MeV, 939.27 MeV, and $126 \mathrm{GeV}$, respectively. The mass formulas also calculate accurately the masses of the new particle at $750 \mathrm{GeV}$ from the LHC and the new light boson at $17 \mathrm{MeV}$. The theoretical base of the accurate mass formulas is the periodic table of elementary particles. As the periodic table of elements is derived from atomic orbitals, the periodic table of elementary particles is derived from the seven principal mass dimensional orbitals and seven auxiliary mass dimensional orbitals. All elementary particles including leptons, quarks, gauge bosons, the Higgs boson, and cosmic rays can be placed in the periodic table of elementary particles.

The periodic table of elementary particles is derived from the theory of everything as the computer simulation model of physical reality consisting of the mathematical computation, digital representation and selective retention components. The mathematical computation involves oscillating M-theory as oscillating membrane-stringparticle whose space-time dimension (D) oscillates between 11D and 10D and between 10D and 4D. For the digital representation component, the three intrinsic data (properties) are rest mass-kinetic energy, electric charge, and spin which are represented by the digital space structure, the digital spin, and the digital electric charge, respectively. The digital representations of rest mass and kinetic energy are 1 as attachment space for the space of matter and 0 as detachment space for the zero-space of matter. The digital representations of the exclusive and the inclusive occupations of positions are 1/2 spin fermion and integer spin boson, respectively. The digital representations of the allowance and the disallowance of irreversible kinetic energy are integral electric charges and fractional electric charges, respectively. For the selective retention component, gravity, the strong 
Table 4. The mass formulas of gauge bosons, cosmic rays, leptons, quarks, and the Higgs boson.

\begin{tabular}{|c|c|c|c|}
\hline $\begin{array}{l}\text { mass } \\
\text { formula of }\end{array}$ & Equation \# & Table \# & Particles involved \\
\hline $\begin{array}{l}\text { gauge } \\
\text { bosons }\end{array}$ & 7 & 1 & $\begin{array}{l}\text { gauge bosons for electromagnetism, strong force, weak force (left), } \mathrm{CP} \\
\text { non-conservation (right), CP non-conservation (left), weak force (right), and gravity }\end{array}$ \\
\hline cosmic rays & 10a. $10 \mathrm{~b}, 11$ & 3 & knees, ankles, and toe \\
\hline leptons & 17 & 2 & electron, muon, tau, and the light boson \\
\hline quarks & 35 & 2 & $\mathrm{~d}, \mathrm{u}, \mathrm{s}, \mathrm{c}, \mathrm{b}$, and $\mathrm{t}$ \\
\hline Higgs boson & 47,48 & & the Higgs boson and the Higgs boson composite \\
\hline
\end{tabular}

force, electromagnetism, and the weak force are the retained events during the reversible four-stage evolution of our universe, and are unified by the common narrative of the evolution. The computer simulation model of physical reality provides the seven principal mass dimensional orbitals and seven auxiliary mass dimensional orbitals to place leptons, quarks, gauge bosons, the Higgs boson, and cosmic rays in the periodic table of elementary particles.

The summary of the mass formulas is in Table 4.

\section{References}

[1] Hansson, J. (2015) International Journal of Modern Physics and Applications, 1, 12-16.

[2] Jammer, M. (2009) Concepts of Mass in Contemporary Physics and Philosophy. Princeton University Press, USA. http://dx.doi.org/10.1515/9781400823789

[3] Chung, D. (1997) Speculations in Science and Technology, 20, 259-268. http://dx.doi.org/10.1023/A:1026433207862

[4] hung, D. (2014) Journal of Modern Physics, 5, 1234-1243. http://dx.doi.org/10.4236/jmp.2014.514123

[5] Chung, D. and Hefferlin, R. (2013) Journal of Modern Physics, 4, 21-26. http://dx.doi.org/10.4236/jmp.2013.44A004

[6] Chung, D. (2014) Journal of Modern Physics, 5, 1467-1472. http://dx.doi.org/10.4236/jmp.2014.515148

[7] Chung, D. (2016) Journal of Modern Physics, 7, 1210-1227. http://dx.doi.org/10.4236/jmp.2016.710110

[8] Chung, D. (2016) Journal of Modern Physics, 7, 642-655. http://dx.doi.org/10.4236/jmp.2016.77064

[9] Woit, P. (2006) Not Even Wrong: The Failure of String Theory and the Search for Unity in Physical Law. Basic Books, New York.

[10] Chung, D. (2015) Journal of Modern Physics, 6, 1820-1832. http://dx.doi.org/10.4236/jmp.2015.613186

[11] Chung, D. and Krasnoholovets, V. (2013) Journal of Modern Physics, 4, 27-31. http://dx.doi.org/10.4236/jmp.2013.44A005

[12] Bounias, M. and Krasnoholovets, V. (2003) The International. Journal of Systems and Cybernetics, 32, $1005-1020$.

[13] Chung, D. (2016) Journal of Modern Physics, 7, 1150-1159. http://dx.doi.org/10.4236/jmp.2016.710104

[14] Tsui, D., Stormer, H. and Gossard, A. (1982) Physical Review Letters, 48, 1559. http://dx.doi.org/10.1103/PhysRevLett.48.1559

[15] Stormer, H. (1999) Reviews of Modern Physics, 71, 875. http://dx.doi.org/10.1103/RevModPhys.71.875

[16] Laughlin, R. (1983) Physical Review Letters, 50, 1395. http://dx.doi.org/10.1103/PhysRevLett.50.1395

[17] Salam, A. (1968) Weak and Electromagnetic Interactions. In: Svartholm, W., Ed., Elementary Particle Theory, Almquist and Wiksell, Stockholm, 367-387.

[18] Drury, L. (2012) Astroparticle Physics, 39-40, 52-60. http://dx.doi.org/10.1016/j.astropartphys.2012.02.006

[19] Sveshnikova, L., Korosteleva, E., Kuzmichev, L., Prosin, V., Ptuskin, V. and Strelnikova, O. (2013) Journal of Physics: Conference Series (JPCS), 409, Article ID: 012062. http://dx.doi.org/10.1088/1742-6596/409/1/012062

[20] Kamilla, R., Wu, X. and Jain, J. (1996) Physical Review Letters, 76, 1332-1335. http://dx.doi.org/10.1103/PhysRevLett.76.1332

[21] Jain, J. (2007) Composite Fermions. Cambridge University Press, New York. http://dx.doi.org/10.1017/CBO9780511607561

[22] Cameron, P. (2011) Apeiron, 18, 29-42. 
[23] MacGregor, M. (2007) The Power of Alpha: The Electron Elementary Particle Generation with Alpha-Quantized Lifetimes and Masses. World Scientific Publishing, Singapore.

[24] Barut, A. (1979) Physical Review Letters, 42, 1251-1253. http://dx.doi.org/10.1103/PhysRevLett.42.1251

[25] Krasznahorkay, A., et al. (2016) Physical Review Letters, 116, Article ID: 042501. http://dx.doi.org/10.1103/PhysRevLett.116.042501

[26] Feng, J., et al. (2016) Physical Review Letters, 117, Article ID: 071803.

[27] De Rujula, A., Georgi, H. and Glashow, S. (1975) Physics Review D, 12, 147. http://dx.doi.org/10.1103/PhysRevD.12.147

[28] Griffiths, D. (2008) Introduction to Elementary Particles. Wiley-VCH, Weinheim, 135.

[29] El Naschie, M. (2002) Chaos, Solitons and Fractals, 14, 369-376. http://dx.doi.org/10.1016/S0960-0779(02)00022-X

[30] Chung, D. (1999) Speculations in Science and Technology, 21, 277-289. http://dx.doi.org/10.1023/A:1005513404873 http://link.springer.com/article/10.1023/A:1005513404873

[31] Chung, D. (2001) arXiv:hep-th/0111147v5.

[32] MacGregor, M. (1990) Il Nuovo Cimento A, 103, 983-1052. http://dx.doi.org/10.1007/BF02782738

[33] Watson, A. (2004) The Quantum Quark. Cambridge University Press, Cambridge, 285-286.

[34] Weinberg, S. (1989) Review Modern Physics, 61, 1-23. http://dx.doi.org/10.1103/RevModPhys.61.1

[35] ATLAS and CMS (2015) CMS PAS EXO-15-004 and ATLAS-CONF-2015-081. https://indico.cern.ch/event/442432/

[36] Nakai, Y., Sato, R. and Tobioka, K. (2016) Physical Review Letters, 116, Article ID: 151802. http://dx.doi.org/10.1103/PhysRevLett.116.151802

[37] The CMS Collaboration (2012) Physics Letters B, 716, 30-61. http://dx.doi.org/10.1016/j.physletb.2012.08.021

[38] The ATLAS Collaboration (2012) Physics Letters B, 716, 1-29. http://dx.doi.org/10.1016/j.physletb.2012.08.020

\section{Submit or recommend next manuscript to SCIRP and we will provide best service for you:}

Accepting pre-submission inquiries through Email, Facebook, LinkedIn, Twitter, etc.

A wide selection of journals (inclusive of 9 subjects, more than 200 journals)

Providing 24-hour high-quality service

User-friendly online submission system

Fair and swift peer-review system

Efficient typesetting and proofreading procedure

Display of the result of downloads and visits, as well as the number of cited articles

Maximum dissemination of your research work

Submit your manuscript at: http://papersubmission.scirp.org/ 